\title{
Analyzing brain structural differences among undergraduates with different grades of self-esteem using multiple anatomical brain network
}

Bo Peng ${ }^{1,2,3}$, Gaofeng Pang ${ }^{4}$, Aditya Saxena ${ }^{5}$, Yan Liu ${ }^{1,2,3}$, Baohua Hu ${ }^{1,2,3}$, Suhong Wang ${ }^{6 *}$ and Yakang Dai ${ }^{1,2,3^{*}}$

${ }^{*}$ Correspondence:

yarmine@gmail.com:

daiyk@sibet.ac.cn

${ }^{1}$ Suzhou Institute

of Biomedical Engineering

and Technology, Chinese

Academy of Sciences,

Suzhou, China

${ }^{6}$ Department of Clinical

Psychology, The Third

Affiliated Hospital

of Soochow University,

Changzhou, China

Full list of author information

is available at the end of the article

\begin{abstract}
Background: Self-esteem is the individual evaluation of oneself. People with high self-esteem grade have mental health and can bravely cope with the threats from the environment. With the development of neuroimaging techniques, researches on cognitive neural mechanisms of self-esteem are increased. Existing methods based on brain morphometry and single-layer brain network cannot characterize the subtle structural differences related to self-esteem.
\end{abstract}

Method: To solve this issue, we proposed a multiple anatomical brain network based on multi-resolution region of interest (ROI) template to study the brain structural connections of self-esteem. The multiple anatomical brain network consists of ROI features and hierarchal brain network features that are extracted from structural MRI. For each layer, we calculated the correlation relationship between pairs of ROIs. In order to solve the high-dimensional problem caused by the large amount of network features, feature selection methods ( $t$-test, mRMR, and SVM-RFE) are adopted to reduce the number of features while retaining discriminative information to the maximum extent. Multi-kernel SVM is employed to integrate the various types of features by appropriate weight coefficient.

Result: The experimental results show that the proposed method can improve classification accuracy to $97.26 \%$ compared with single-layer brain network.

Conclusions: The proposed method provides a new perspective for the analysis of brain structural differences of self-esteem, which also has potential guiding significance in other researches involved brain cognitive activity and brain disease diagnosis.

Keywords: Structural magnetic resonance imaging, Self-esteem, Multi-resolution ROI, Hierarchal brain network, Machine learning method

\section{Background}

Self-esteem is regarded as self-affirmation and self-identification about oneself [1]. People with good mental health have a higher self-esteem grade and think of themselves as valuable persons [2]. Researchers found that undergraduates with different self-esteem 
grades have brain structural differences. These people feel that they deserve to be respected by others, and are more able to accept individual's deficiencies [3]. However, people with low self-esteem have low self-confidence, and the outside world will have a great impact on them, resulting in low socioeconomic status and poor physical health. Neurophysiology researchers found that self-esteem may be composed of multiple subsystems that are structurally separated from each other, but functionally interact [4]. Brain imaging studies suggest that self-esteem involves multiple psychological processes, including self in perception, memory, and introspection. These psychological processes have their own corresponding brain regions. For example, self-face recognition occurs in right brain, and autobiographical memory is mainly related to hippocampus, and self-reference is related to medial prefrontal lobe [5]. In addition to these independent brain regions, the difference in self-esteem is also reflected in brain network connection. Medial prefrontal cortex is activated during the process of social, self, and affective events [6]. Therefore, in this study, we focus on exploring the brain structural differences among undergraduates with different levels of self-esteem.

Brain network aims to study the interaction of various brain regions as a whole, which has an important role to deeply understand brain structures and cognitive neural processes. The anatomical brain network mainly uses region of interest (ROI) of the brain as the node, and the correlation between brain regions as edge [7, 8]. The definition of ROI is a key step in anatomical brain network analysis. Most existing methods use ROIbased brain network analysis methods to study brain structure and functional connections related to self-esteem. Kelly et al. use the cerebral blood flow imaging method to estimate the hemodynamic response function of each ROI, in order to study the brain networks that are activated during the processing of self-esteem related information [9]. Goldin et al. used functional magnetic resonance imaging (fMRI) technology to measure changes in the brain network between self-esteem group and the self-confidence group by measuring the BOLD response in the ROI [10]. Chavez et al. conducted a psychophysiological interaction analysis to calculate the correlation between specific ROIs related to self-esteem [11]. Although a variety of neuroimaging methods can be used to explore the cognitive mechanism of the brain, structural magnetic resonance imaging (sMRI) is widely used in the analysis of brain anatomical networks due to its high resolution of brain soft tissue imaging [12]. Studies based on sMRI show that self-esteem involves multiple networks related to self-reference processing, autobiographical memory, and social cognition, including default mode networks and social cognition networks [13]. In addition, self-esteem shows the brain network mechanism dominated by bilateral brain and mainly controlled by right brain [14]. Although the above researches have initially revealed the brain network representation of self-esteem, it only used single-layer network that cannot fully identify the subtle differences in network connectivity caused by self-esteem.

The motivation of this study is to use enhanced feature representation method to better analyze brain structural connectivity related to self-esteem. In recent years, machine learning techniques become a research hotspot in the field of brain network analysis due to its ability to learn pattern from data and predict unknown data [8]. Brain network analysis can help us fully understand the cognitive psychological activity of selfesteem. However, there are few studies using machine learning methods to construct 
self-esteem-related brain network, especially for the construction of hierarchal brain networks. In this article, we propose multiple anatomical brain network construction method based on multi-resolution ROIs. The innovation of this method is to use the in-layer and between-layer connections to better describe the correlation between small brain regions and large brain functional areas, which improves the feature expression ability of single-layer brain network.

\section{Results}

\section{Classification performance}

Various indexes can be used to evaluate the classification performance of the proposed method. The evaluation indicators including accuracy (ACC), sensitivity (SEN), specificity (SPE), area under the receiver operating characteristic curve (AUC), $F$ score, balanced accuracy, Youden's index are listed in Table 1. The results show that the multiple brain network features have the highest classification accuracy of $97.26 \%$, and the AUC is also greater than other feature types. This indicates that the multiple brain network features have advantages in characterizing structural differences at the global level. In addition, the higher specificity and sensitivity also show that the multiple brain network features have better recognition capabilities in exploring the subtle differences in brain structure caused by self-esteem (Fig. 1).

\section{Weight coefficient}

The role of the weight coefficient is to determine the proportion of the various types of features in the multi-kernel classifier (Fig. 2). Appropriate weight coefficient helps achieve the best classifier performance. A smaller weight coefficient indicates that the contribution of ROI features is lower, while the contribution of the hierarchical brain network features is higher. Through experiments, we can find the most suitable weight coefficient in the range of $0-1$.

The weight coefficient has an important influence on the performance of the classifier. It is proved that the weight coefficient makes the classifier perform well in the relatively large range from 0.05 to 0.35 , which can decline the difficulty of determining the ratio of the two types of features, which reflects the robustness of our proposed method. The best results are obtained at 0.05 . At this time, the hierarchical

Table 1 Classification performance using different feature types

\begin{tabular}{|c|c|c|c|c|c|c|c|}
\hline & $\mathrm{ACC}(\%)$ & AUC (\%) & SEN (\%) & SPE (\%) & $Y(\%)$ & $F(\%)$ & BAC (\%) \\
\hline Network features in the 4 th layer & 90.69 & 96.63 & 87.72 & 90.65 & 86.74 & 77.38 & 88.77 \\
\hline Network features in the 3 rd layer & 88.31 & 84.27 & 85.32 & 84.29 & 88.33 & 82.62 & 85.74 \\
\hline Network features in the 2 nd layer & 89.59 & 76.65 & 78.53 & 75.94 & 73.24 & 69.18 & 67.77 \\
\hline Network features in all layers & 92.59 & 91.93 & 91.51 & 90.91 & 93.27 & 87.18 & 91.49 \\
\hline $\mathrm{ROI}$ features in the 4th layer & 88.69 & 85.63 & 87.72 & 87.65 & 86.74 & 77.38 & 88.77 \\
\hline $\begin{array}{l}\text { ROI features and network features in the } \\
\text { 4th layer }\end{array}$ & 94.41 & 95.58 & 94.42 & 93.41 & 92.47 & 92.82 & 92.64 \\
\hline $\begin{array}{l}\text { Multilevel (ROI features in the 4th layer and } \\
\text { network features in all layers) }\end{array}$ & 97.26 & 99.88 & 97.27 & 97.41 & 97.12 & 94.53 & 97.27 \\
\hline
\end{tabular}

ACC: accuracy; AUC: area under receiver operating characteristic curve; SEN: sensitivity; SPE: specificity; Y:Youden's index; F:F-score; BAC: balanced accuracy 


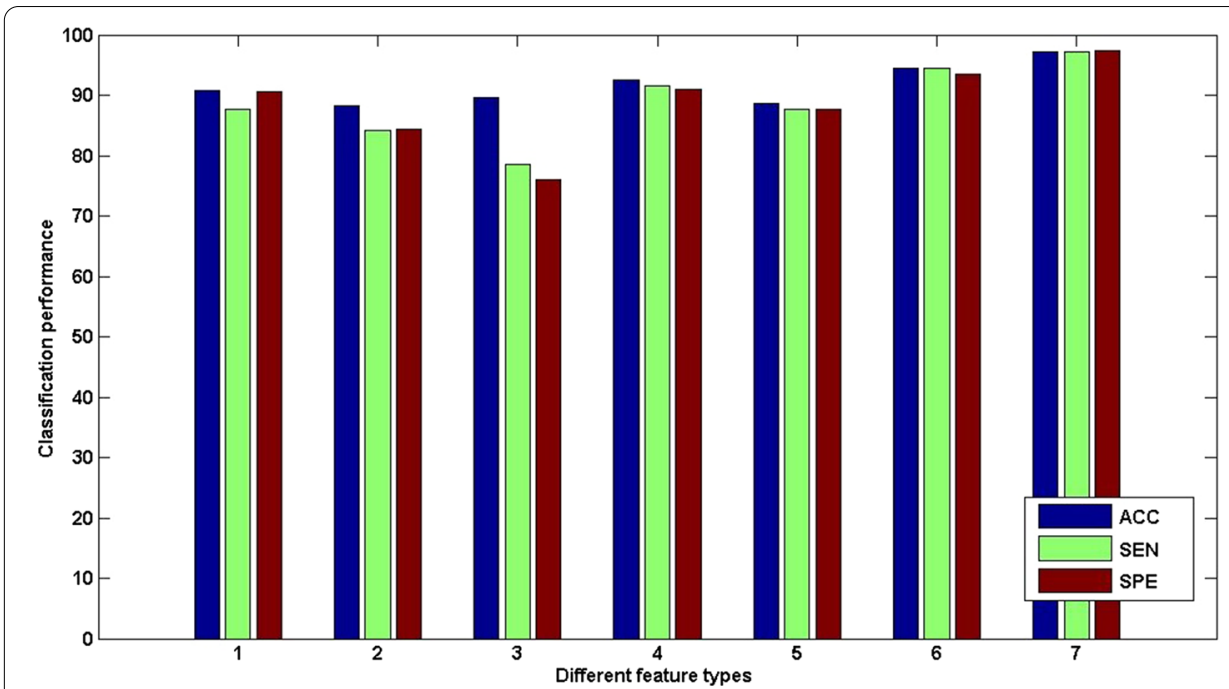

Fig. 1 Boxplot of classification accuracy for different feature types. (1) Network features in the 4th layer; (2) network features in the 3rd layer; (3) network features in the 2nd layer; (4) network features in all layers; (5) $\mathrm{ROI}$ features in the 4th layer; (6) ROI features and network features in the 4th layer; (7) multilevel features. ACC accuracy, SEN sensitivity, SPE specificity

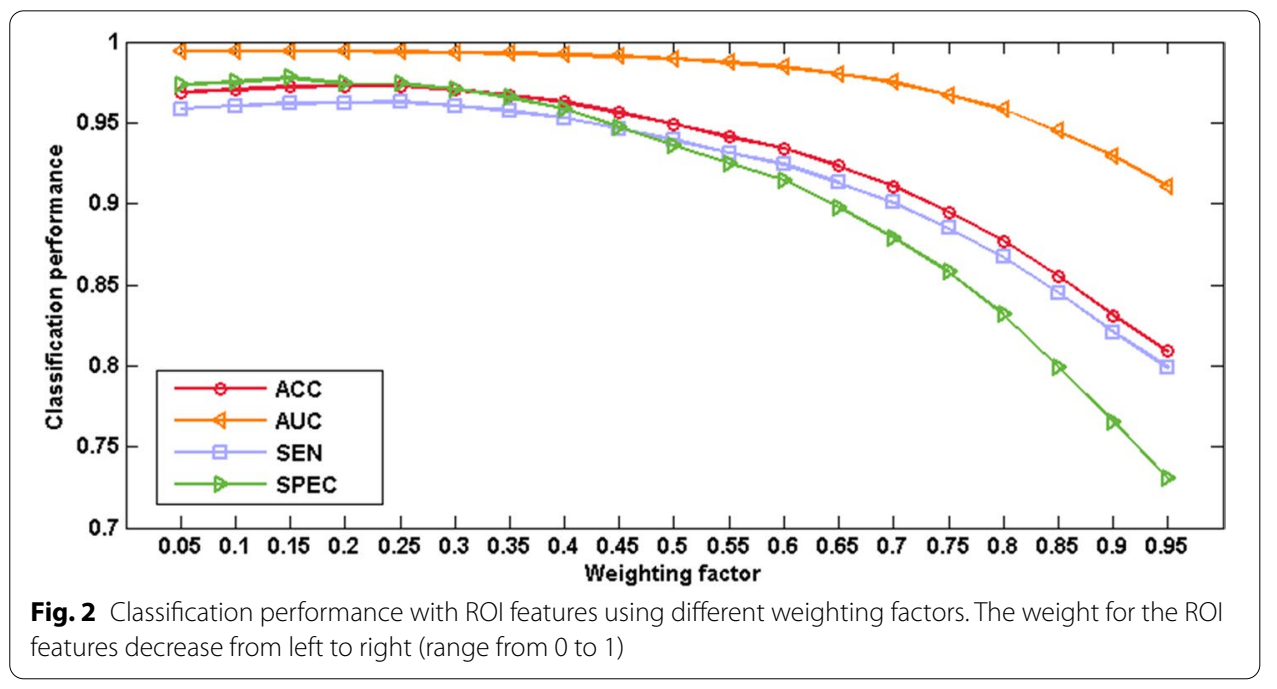

brain network features contributed more to the classification than the ROI features. This is because the hierarchical brain network can fully express the differences in brain structure between the high self-esteem group and low self-esteem group.

\section{Top discriminative features}

We use the proposed method to select the most discriminative ROI features (Fig. 3). These ROIs include occipital lobe (superior and middle occipital gyrus, cuneus), frontal lobe (supplementary motor area, middle frontal gyrus), temporal lobe (middle temporal gyrus), parietal lobe (precuneus, angular gyrus), limbic lobe (posterior cingulate gyrus), and central region (precentral gyrus). The experimental results also 


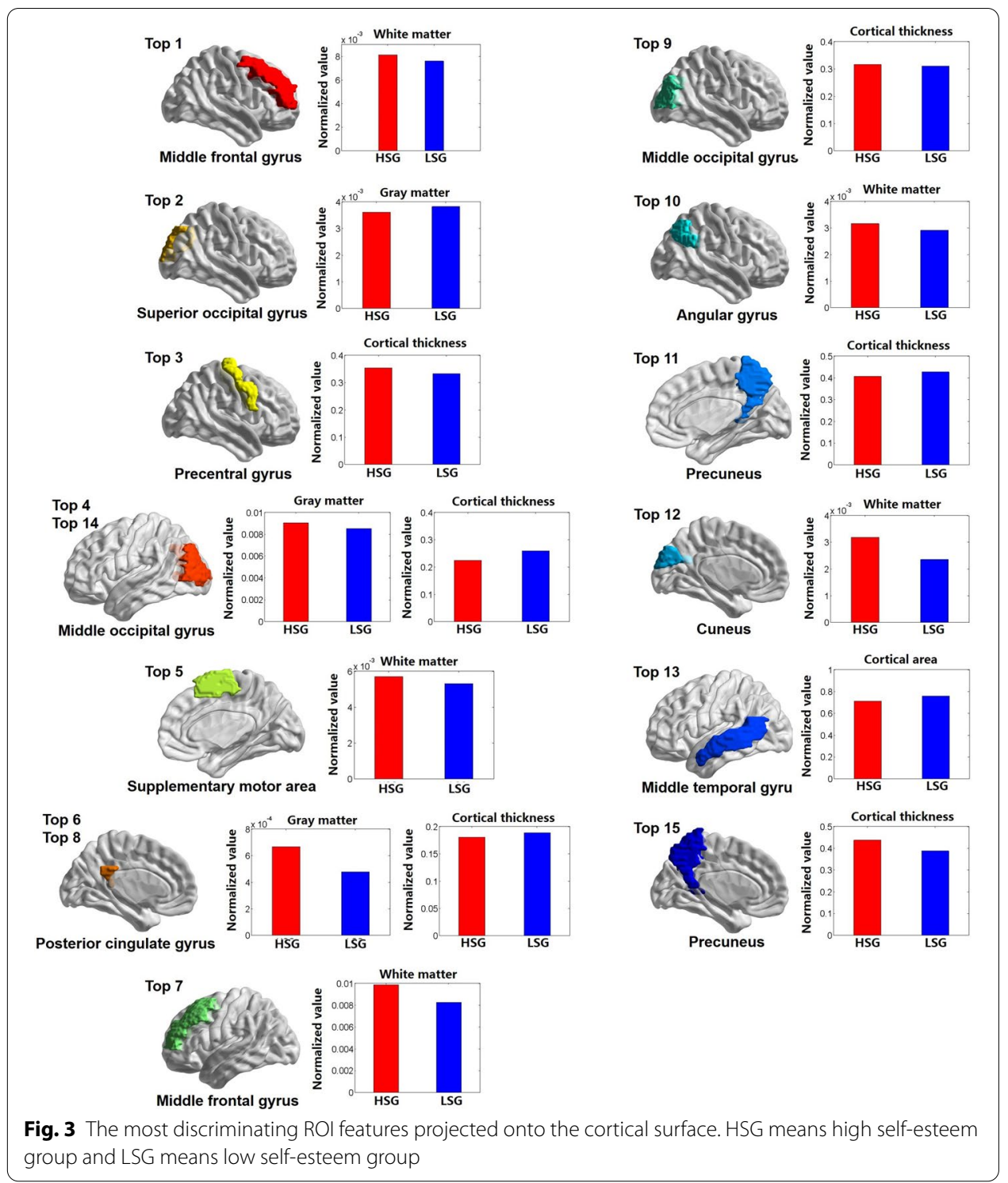

show that differences in brain structure related to self-esteem are mainly in white matter and cortical thickness (Table 2).

The top 15 network features selected from all four layers (Table 3). The most discriminative hierarchical network features are mainly distributed in limbic lobe and parietal lobe (Fig. 4).

\section{Discussion}

We studied multiple anatomical brain network related to self-esteem. Our results demonstrate that the proposed method is superior to the single-layer network method. The multiple networks enhance the representation of the specific brain structure related to self-esteem, thereby providing an effective and novel method to detect self-esteemrelated biomarkers. 
Table 2 Top 15 most discriminating regional features that were selected using the proposed classification framework

\begin{tabular}{llllll}
\hline No. & Name of ROI & L/R & Tissue & Brain lobe & Frequency \\
\hline 1 & Middle frontal gyrus & $\mathrm{R}$ & WM & Frontal lobe & 185 \\
2 & Superior occipital gyrus & $\mathrm{R}$ & GM & Occipital lobe & 144 \\
3 & Precentral gyrus & $\mathrm{R}$ & Thickness & Central region & 141 \\
4 & Middle occipital gyrus & $\mathrm{L}$ & GM & Occipital lobe & 102 \\
5 & Supplementary motor area & $\mathrm{R}$ & WM & Frontal lobe & 86 \\
6 & Posterior cingulate gyrus & $\mathrm{L}$ & CSF & Limbic lobe & 75 \\
7 & Middle frontal gyrus & $\mathrm{L}$ & WM & Frontal lobe & 73 \\
8 & Posterior cingulate gyrus & $\mathrm{L}$ & Thickness & Limbic lobe & 70 \\
9 & Middle occipital gyrus & $\mathrm{R}$ & Thickness & Occipital lobe & 68 \\
10 & Angular gyrus & $\mathrm{R}$ & WM & Parietal lobe & 64 \\
11 & Precuneus & $\mathrm{R}$ & Thickness & Parietal lobe & 58 \\
12 & Cuneus & $\mathrm{L}$ & WM & Occipital lobe & 58 \\
13 & Middle temporal gyrus & $\mathrm{L}$ & Area & Temporal lobe & 54 \\
14 & Precuneus & $\mathrm{L}$ & Thickness & Parietal lobe & 53 \\
15 & Middle occipital gyrus & $\mathrm{L}$ & Thickness & Occipital lobe & 53 \\
\hline
\end{tabular}

L: left hemisphere; R: right hemisphere; GM: gray matter volume; WM: white matter volume; CSF: cerebrospinal volume; Thickness: cortical thickness; Area:cortical surface area; Frequency: selected frequency over 100 repetitions of twofold crossvalidation

Table 3 Top 15 similarity features that were selected using the proposed classification framework

\begin{tabular}{|c|c|c|c|c|c|c|}
\hline Network & Name of ROI & $\mathrm{L} / \mathrm{R}$ & Name of ROI & L/R & No. & Frequency \\
\hline \multirow[t]{4}{*}{ Network 4} & Orbitofrontal cortex (inferior) & $L$ & Superior parietal gyrus & $L$ & 15 & 45 \\
\hline & Rectus gyrus & $L$ & Precuneus & $L$ & 12 & 48 \\
\hline & Orbitofrontal cortex (inferior) & $\mathrm{L}$ & Paracentral lobule & $\mathrm{R}$ & 10 & 54 \\
\hline & Orbitofrontal cortex (inferior) & $\mathrm{R}$ & Precuneus & $L$ & 3 & 95 \\
\hline \multirow[t]{9}{*}{ Network 3} & Parietal lobe: lateral surface & $\mathrm{R}$ & $\begin{array}{l}\text { Limbic lobe: Temporal pole } \\
\text { (superior) }\end{array}$ & $\mathrm{R}$ & 1 & 118 \\
\hline & Frontal lobe: lateral surface & $\mathrm{L}$ & Parietal lobe: lateral surface & $L$ & 2 & 114 \\
\hline & Temporal lobe: lateral surface & $L$ & Parietal lobe: lateral surface & $\mathrm{R}$ & 4 & 93 \\
\hline & Frontal lobe: lateral surface & $\mathrm{R}$ & Temporal lobe: lateral surface & $\mathrm{R}$ & 5 & 93 \\
\hline & Frontal lobe: lateral surface & $\mathrm{R}$ & Parietal lobe: lateral surface & $L$ & 6 & 92 \\
\hline & $\begin{array}{l}\text { Central region: Rolandic oper- } \\
\text { culum }\end{array}$ & $L$ & $\begin{array}{l}\text { Limbic lobe: temporal pole } \\
\text { (superior) }\end{array}$ & $\mathrm{L}$ & 7 & 74 \\
\hline & Central region: postcentral gyrus & $\mathrm{R}$ & Parietal lobe: lateral surface & $\mathrm{R}$ & 9 & 61 \\
\hline & Temporal lobe: lateral surface & $\mathrm{R}$ & $\begin{array}{l}\text { Limbic lobe: temporal pole } \\
\text { (superior) }\end{array}$ & $\mathrm{R}$ & 13 & 47 \\
\hline & Parietal lobe: lateral surface & $L$ & $\begin{array}{l}\text { Limbic lobe: temporal pole } \\
\text { (superior) }\end{array}$ & $L$ & 14 & 47 \\
\hline \multirow[t]{2}{*}{ Network 2} & Central region & $L$ & Limbic lobe & $L$ & 8 & 73 \\
\hline & Central region & $\mathrm{R}$ & Limbic lobe & $\mathrm{L}$ & 11 & 54 \\
\hline
\end{tabular}

L: left hemisphere; R: right hemisphere; Frequency:selected frequency over 100 repetitions of twofold cross-validation

\section{Improvement of the proposed method}

It is difficult to fully understand the functional organization of the brain using only a single-layer network framework since the brain is a complex system. In this study, we construct a multiple anatomical brain network in multi-resolution ROIs to 

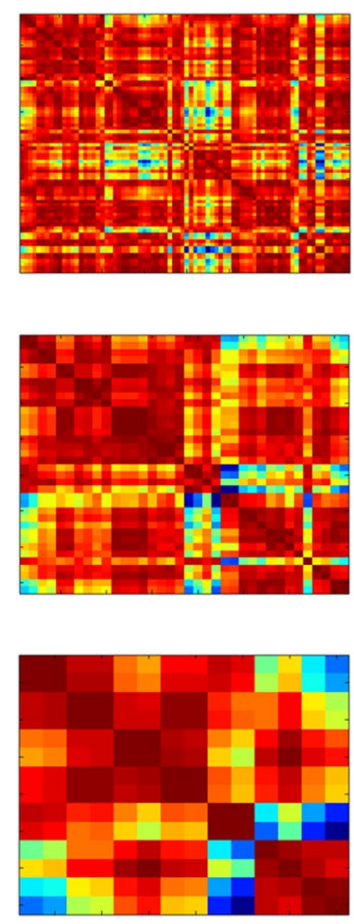
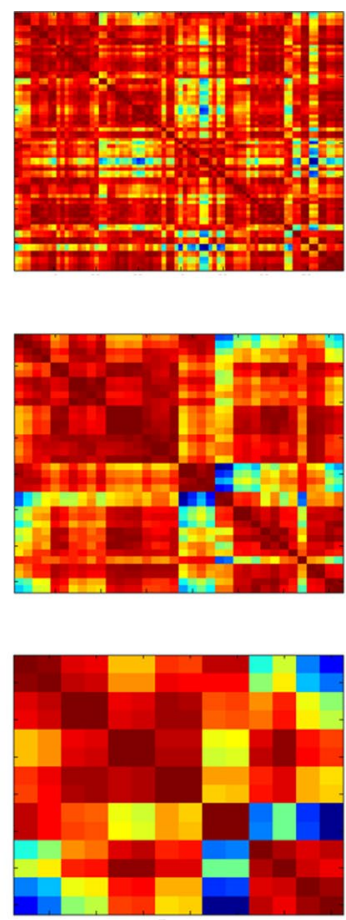

b
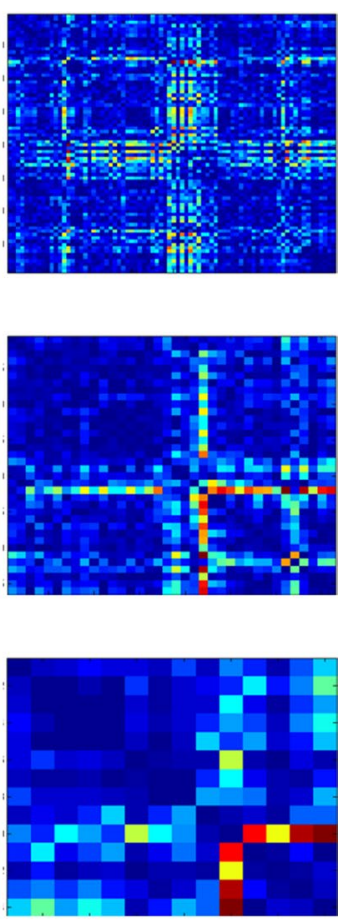

c

Fig. 4 Correlative matrix: a high self-esteem group, b low self-esteem group, $\mathbf{c}$ differences between the two groups

improve the classification performance. Compared with the single-layer networkbased method, multiple networks enhance the classification performance by using supplementary information from different networks. Compared with the best results obtained using a single-layer network, our proposed method can improve the classification accuracy by $8.95 \%$ (Table 1 ).

\section{Analysis of discriminative features}

The discriminative ROI features discovered by our method are distributed in multiple regions of the brain. Because few current studies employ automatic classification method to study the brain structure of self-esteem, we only compare brain regions found through our machine learning method with existing morphological based studies. Compared with previous studies, our results showed consistency in departmental brain regions, including precuneus [4], precentral gyrus [15], middle frontal gyrus [16], cuneus [4], posterior cingulate [17]. This indicates the effectiveness of our classification method in revealing brain regions related to self-esteem. In addition to these consistent regions, we also found that the middle occipital, superior occipital, and supplementary motor are related to self-esteem. These brain regions have not been reported in previous studies, which is worth paying attention to in the follow-up study.

The discriminative network features are mainly located on frontal, parietal and limbic lobe. After a comprehensive analysis of existing research on neuropsychological mechanisms related to self-esteem, we found that the frontal region is an important part of the neural basis related to self-esteem. The frontal lobe is responsible for self-evaluation, 
self-regulation, and emotion management. Individuals with low self-esteem have a stronger emotional response to social evaluations, while high self-esteem individuals show stronger self-positivity in the process of self-evaluation. These findings indicate that frontal lobe plays an important role in generating positive self-information.

\section{Comparison with other methods}

Since few studies have used machine learning to analyze the relationship between selfesteem and brain structure, we compare our results with the current morphological studies related to self-esteem. At present, most studies have found a correlation between self-esteem and frontal lobe $[6,16,17]$. The frontal lobe is mainly responsible for the cognitive activities of the brain, and self-esteem involves cognitive processing and emotional response. The results of this paper are generally consistent with those of previous studies. In addition, some specific brain regions, such as the cuneus, have been found to be related to many self-related functions, such as self-related information processing and various aspects of consciousness [16]. In addition, we also found a correlation between cingulate cortex and self-esteem. Studies have shown that when individuals are accepted by society, individuals activate the ventral anterior cingulate cortex and medial prefrontal cortex, thus enhancing self-esteem [17]. Therefore, the study of brain structure with different self-esteem grades is helpful to understand the neurophysiological mechanism of self-esteem.

At the same time, in order to prove the effectiveness of the proposed method. We have compared the classification performance with different classifiers. Table 4 shows the classification results using different classifies. The results show that the proposed method based on multiple anatomical brain networks has better performance than all the other classifiers.

\section{Limitations and future directions}

Although the classification performance is good, our study still has some limitations. Here, we put forward some future directions in order to conduct a better research on self-esteem in cognitive neuroscience. First, as a preliminary study, we use relatively small amount of data in machine learning. In the follow-up study, we will collect some more data to get more reasonable analysis and make more detailed grades of self-esteem, taking psychological, individual, and social factors into account. Second, other metrics for modeling the interactions between ROIs, such as Euclidean distance and the L1norm distance measure. Third, with the increasing of the data, deep learning can also

Table 4 The classification results using different classifies

\begin{tabular}{ll}
\hline Configurations & $\begin{array}{l}\text { Classification } \\
\text { accuracy (\%) }\end{array}$ \\
\hline SVM (RBF) & 97.27 \\
SVM (linear) & 97.07 \\
K-nearest neighbor classifier & 94.25 \\
Naive Bayes classifier & 92.13 \\
Decision-tree algorithm & 91.19 \\
\hline
\end{tabular}


be used to automatically extract features to find the discriminative features of multiple brain network, such as depth automatic encoder. Fourth, due to the multi-types of features involved in this study, multiple weight factors can be used for better feature fusion.

\section{Conclusion}

In this study, we have presented how multiple anatomical brain networks can be used to analyze brain structural differences among undergraduates with different grades of self-esteem. Several feature selection methods are adopted to reduce the number of features, and multi-kernel SVM was employed to integrate various types of features by appropriate weight coefficient. The features extracted from these networks can be used to improve the defects that the traditional single-layer brain network contains insufficient information. The experiments show that our method has improved performance compared with the single-layer network structure, which can provide a new perspective for the analysis of brain structure differences of self-esteem. It also has potential guiding significance of out method in other researches involved in brain cognitive activity and brain disease diagnosis.

\section{Methods}

\section{Subjects}

The structural MRI data used in our study were acquired from Soochow University, which is composed of 68 undergraduates. The study was approved by the Ethics Committee of the Third Affiliated Hospital of Soochow University. Written informed consents were obtained from all subjects. All subjects did not receive stimulants or hypnotics before acquisition in order to keep them awake and let the brain work normally. All participants' vision were normal or corrected to normal, and they were right-handed. After the test, each participant will receive a small gift or financial reward. All subjects are required to perform Rosenberg Self-esteem Scale (RSES) test. The RSES is originally developed by Rosenberg in 1965 to assess the overall feelings of undergraduates about self-worth and self-acceptance. It is the most used self-esteem measurement tool in the psychology community [18]. We ranked the RSES test scores from highest to lowest, and then divided them into two groups: high self-esteem group and low self-esteem group. Table 5 provides detailed information of all participants.

Table 5 Demographic information of all subjects

\begin{tabular}{lllc}
\hline & High self-esteem group & Low self-esteem group & $\boldsymbol{p}$ value \\
\hline Subjects & 34 & 34 & \\
Male/female & $19 / 15$ & $16 / 18$ & 0.83 \\
Age (mean \pm SD) & $21.90 \pm 1.16$ & $22.53 \pm 1.42$ & 0.77 \\
Rosenberg Scale (mean \pm SD) & $25.35 \pm 0.81$ & $17.86 \pm 3.35$ & $<0.001$ \\
\hline
\end{tabular}

The $p$-value of gender was obtained by Chi-squared test

The $p$-values of age and Rosenberg scale were obtained by $t$-test

Significance level was set to 0.05 


\section{Imaging acquisition and preprocessing}

All images were collected on a 3T Siemens Medical Systems equipment. The acquisition parameters are set as: echo time $(\mathrm{TE})=2.98 \mathrm{~ms}$, repetition time $(\mathrm{TR})=2300 \mathrm{~ms}$, flip angle $(\mathrm{FA})=9^{\circ}$, voxel size $=1 \times 1 \times 1 \mathrm{~mm}^{3}$, slice thickness $=1 \mathrm{~mm}$, field of view $(\mathrm{FoV})=256 \mathrm{~mm}$.

We use an automatic pipeline for sMRI image processing. Firstly, we adjusted the image orientation (axial, coronal, and sagittal) to match the template image, and performed offset field correction to remove the gray-scale unevenness of the image [19]. Secondly, the brain image was extracted by removing the skull and cerebellum [20]. Thirdly, gray matter (GM), white matter (WM) and cerebrospinal fluid (CSF) were segmented from the background [21]. Fourth, the segmented image was registered to the template labeled with the Automated Anatomical Labeling (AAL) template [22]. Fifth, in order to calculate the morphological features based on the cortex, the middle layer of the cerebral cortex was depicted [23]. After the whole processing, the morphological measurements of GM volume, WM volume, CSF volume, cortical thickness, and cortical surface area of each ROI were obtained for each subject. It should be noted that we removed 12 subcortical ROIs from AAL template considering that the cerebral cortex contains more neurons.

\section{Framework of the proposed method}

The framework of the proposed method based on multiple brain network is shown in Fig. 5, mainly including image processing, feature extraction, feature selection, and classification. Multiple brain network were constructed based on morphological features (volume of different brain tissue, cortical thickness, and cortical surface area). Feature selection can reduce the dimensionality of high-dimensional brain network features, only retaining the features that can maximize the specificity of the subjects. The optimal feature subset can be trained by the classifier as neuroimaging markers representing different self-esteem levels.

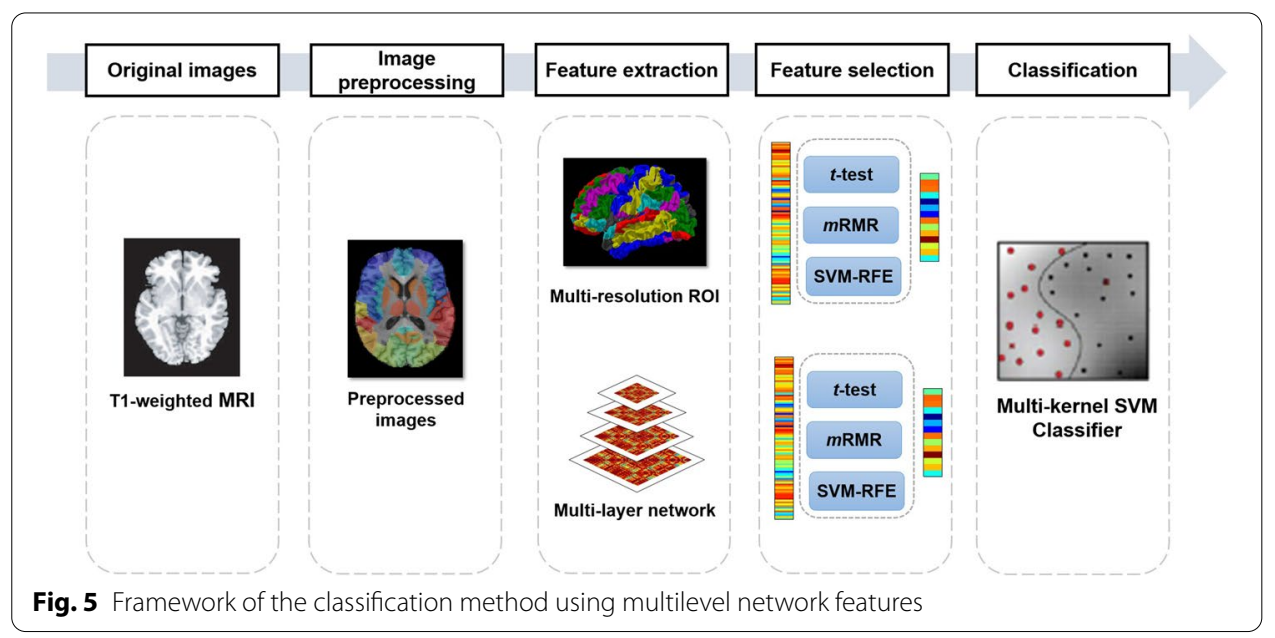




\section{Construction of multiple anatomical networks}

Through the above image processing steps, GM volume, WM volume, CSF volume, cortical thickness, and cortical surface area of each ROI can be obtained from the MRI image of each subject. In order to reduce individual differences, standardization was performed, dividing the measured value of each ROI by the total intracranial volume, mean cortical thickness, and whole cerebral cortical surface area of the subject. Therefore, we used normalized volume and cortical features to provide a more appropriate representation. More objective measurements can be received by such processing. In order to improve the performance of the classifier, we propose a four-layer hierarchical network in this paper. We used brain templates with different ROI resolution in each layer to construct brain network nodes and edges.

Specifically, the bottommost template containing 78 ROIs is defined as $L^{4}$, the remaining three layers are defined as $L^{l}$, where $l=1,2,3$. A larger $l$ value indicates a higherresolution ROI, which is located in the brain network layer closer to the bottom of the hierarchy. By merging small brain regions into large brain functional areas, the number of ROIs is reduced. In the layer $L^{3}$, there are 36 ROIs by dividing the whole brain into lateral, medial and inferior surfaces. In the layer $L^{2}, 14$ ROIs are defined reefing to the anatomical brain structure of central, frontal, parietal, occipital, temporal, limbic, and insula lobe. The specific definition rules of these ROIs can be found in Table 6. It is worth noting that in the first layer $L^{1}$, we study the brain as a whole.

For each layer, correlation between ROIs can be calculated using brain template defined above. Its node correspond to the ROIs in different resolution, and the edge corresponds to the interaction between pairs of ROIs. Take the bottom layer $L^{4}$ as an example, a $78 \times 78$ matrix $C^{4}$ can be calculated by computing the Pearson correlation coefficient between the $i$ th ROI and $j$ th ROI. We define

$$
C^{4}(i, j)=\exp \left\{-\frac{[t(i)-t(j)]^{2}}{2 \sigma^{2}}\right\},
$$

where $t(i)$ and $t(j)$ represent the mean thickness of the cerebral cortex corresponding to the $i$ th and $j$ th ROIs.

$\sigma$ is defined as $\sigma=\sqrt{\sigma_{i}^{2}+\sigma_{j}^{2}}$, where $\sigma_{i}$ and $\sigma_{j}$ represent the standard deviation of cortex for the $i$ th and $j$ th ROI. Due to the symmetry of the correlation matrix, we only use the upper triangular elements of the matrix $C^{4}$ to construct the feature vector. We connect the 3003 upper triangular elements to form the corresponding feature vector for $L^{4}$. Since the ROIs in the remaining three layers are obtained by merging ROIs in the bottommost layer, the mean and standard deviation of these compound ROIs can be obtained by calculating the average value of all ROIs. The definition of correlation matrix $C^{l}$ for other layers is similar to $C^{4}$. The union of the hierarchical networks is constructed by junction of the four upper triangular correlation matrix into a long vector.

\section{Feature selection}

In order to reduce the feature dimension and filter out the most discriminative features, we adopted several feature selection methods. First, we preliminarily select the features by comparing the statistics of different features. The statistical $t$-test $(p<0.05)$ is adopted 
Table 6 Regions of interest (ROIs) defined in the automated anatomical labeling (AAL) template

\begin{tabular}{|c|c|c|c|c|c|}
\hline \multicolumn{2}{|c|}{ Network 2} & \multicolumn{2}{|c|}{ Network 3} & \multicolumn{2}{|c|}{ Network 4} \\
\hline No. & Name of ROI & No. & Name of ROI & No. & Name of ROI \\
\hline \multirow[t]{3}{*}{1,2} & Central region & 1,2 & Central region: precentral gyrus & 1,2 & Precentral gyrus \\
\hline & & 3,4 & Central region: postcentral gyrus & 53,54 & Postcentral gyrus \\
\hline & & 5,6 & Central region: Rolandic operculum & 17,18 & Rolandic operculum left \\
\hline \multirow[t]{13}{*}{3,4} & Frontal lobe & 7,8 & Frontal lobe: lateral surface & 3,4 & Superior frontal gyrus (dorsal) \\
\hline & & & & 7,8 & Middle frontal gyrus \\
\hline & & & & 11,12 & Inferior frontal gyrus (opercular) \\
\hline & & & & 13,14 & Inferior frontal gyrus (triangular) \\
\hline & & 9,10 & Frontal lobe: medial surface & 19,20 & Supplementary motor area \\
\hline & & & & 23,24 & Superior frontal gyrus (medial) \\
\hline & & & & 65,66 & Paracentral lobule \\
\hline & & 11,12 & Frontal lobe: orbital surface & 5,6 & Orbitofrontal cortex (superior) \\
\hline & & & & 9,10 & Orbitofrontal cortex (middle) \\
\hline & & & & 15,16 & Orbitofrontal cortex (inferior) \\
\hline & & & & 21,22 & Olfactory \\
\hline & & & & 25,26 & Orbitofrontal cortex (medial) \\
\hline & & & & 27,28 & Rectus gyrus \\
\hline \multirow[t]{4}{*}{5,6} & Temporal lobe & 13,14 & Temporal lobe: lateral surface & 67,68 & Heschl gyrus \\
\hline & & & & 69,70 & Superior temporal gyrus \\
\hline & & & & 73,74 & Middle temporal gyrus \\
\hline & & & & 77,78 & Inferior temporal gyrus \\
\hline \multirow[t]{5}{*}{7,8} & Parietal lobe & 15,16 & Parietal lobe: lateral surface & 55,56 & Superior parietal gyrus \\
\hline & & & & 57,58 & Inferior parietal lobule \\
\hline & & & & 59,60 & Supramarginal gyrus \\
\hline & & & & 61,62 & Angular gyrus \\
\hline & & 17,18 & Parietal lobe: medial surface & 63,64 & Precuneus \\
\hline \multirow[t]{7}{*}{9,10} & Occipital lobe & 19,20 & Occipital lobe: lateral surface & 45,46 & Superior occipital gyrus \\
\hline & & & & 47,48 & Middle occipital gyrus \\
\hline & & & & 49,50 & Inferior occipital gyrus \\
\hline & & 21,22 & Occipital lobe: medial and inferior & 39,40 & Calcarine cortex \\
\hline & & & & 41,42 & Cuneus \\
\hline & & & & 43,44 & Lingual gyrus \\
\hline & & & & 51,52 & Fusiform gyrus \\
\hline \multirow[t]{6}{*}{11,12} & Limbic lobe & 23,24 & Limbic lobe: temporal pole (superior) & 71,72 & Temporal pole (superior) \\
\hline & & 25,26 & Limbic lobe: temporal pole (middle) & 75,76 & Temporal pole (middle) \\
\hline & & 27,28 & Limbic lobe: anterior cingulate gyrus & 31,32 & Anterior cingulate gyrus \\
\hline & & 29,30 & Limbic lobe: middle cingulate gyrus & 33,34 & Middle cingulate gyrus \\
\hline & & 31,32 & Limbic lobe: posterior cingulate gyrus & 35,36 & Posterior cingulate gyrus \\
\hline & & 33,34 & Limbic lobe: Parahippocampal gyrus & 37,38 & Parahippocampal gyrus \\
\hline 13,14 & Insula & 35,36 & Insula: insula & 29,30 & Insula \\
\hline
\end{tabular}

to remove features with small differences (the features with small differences are difficult to distinguish the two groups). Then, another filter-based feature selection method called minimum redundancy and maximum correlation (mRMR) is used to remove the redundant features [24]. The core idea of mRMR is to maximize the correlation between features and classification variables, and minimize the correlation between different 
features. After the above two filter-based feature selections, the machine learning recursive feature elimination (SVM-RFE) method [25] is used to further reduce the feature dimension. SVM-RFE is proposed in classification of cancer, and has good performance and strong generalization ability. It is the combination of SVM and subsequent search strategy. It trains samples through the model, and then ranks the scores of each feature to remove the feature with the smallest score, and then trains the model again with the remaining features for the next iteration, and finally selects the number of features that are needed. After completing the entire feature selection steps, the optimal feature subset is obtained.

\title{
Classification using multi-kernel SVM
}

There are various types of features in the multiple brain network, one is the high-resolution ROI features in the fourth layer, and the other is the brain network features corresponding to different layers. Multi-kernel machine learning method can integrate these various types of features into a final classifier. Firstly, a Gaussian Radial Basis Function (RBF) kernel function is used to construct a kernel matrix for each type of feature. Secondly, the two kernel matrices are integrated into the multi-kernel matrix through appropriate weight coefficients [25]. Comparing the results of using linear kernel function and using RBF function (non-linear), we discover that the RBF kernel can significantly improve the classification performance. Therefore, we choose the RBF kernel function to construct the multi-kernel classifier. Finally, the optimal features subset can be obtained.

\section{Cross-validation}

The nested cross-validation method has been applied in our previous research [26]. In the inner loop, the training set are used to determine the parameters of the classifier. In the outer loop, the testing set is used to evaluate the generalization ability of the classifier. It should be noted that at the beginning of the experiment, the entire data set was randomly divided into two parts, one for training and the other one for testing. The training set and testing set can be exchanged throughout the verification process, while the processing steps remain unchanged.

\begin{abstract}
Abbreviations
ROI: Region of interest; fMRI: Functional magnetic resonance imaging; sMRI: Structural magnetic resonance imaging; RSES: Rosenberg Self-esteem Scale; TE: Echo time; TR: Repetition time; FA: Flip angle; FoV: Field of view; GM: Gray matter; WM: White matter; CSF: Cerebrospinal fluid; AAL: Automated Anatomical Labeling; mRMR: The minimum redundancy and maximum correlation; SVM-RFE: The machine learning recursive feature elimination; RBF: Radial Basis Function.
\end{abstract}

\section{Acknowledgements}

The authors would like to thank the physicians in department of neuroscience, the Third Affiliated Hospital of Soochow University for their help collecting the brain MR images of the young adults with different self-esteem scales.

\section{Authors' contributions}

SW and GP contributed to the conception of the study. AS and JW performed the experiment. YD and BP contributed significantly to analysis and manuscript preparation. BP performed the data analyses and wrote the manuscript. BP and $\mathrm{CP}$ performed the analysis with constructive discussions. All authors read and approved the final manuscript.

\section{Funding}

This work was supported by National Key Research and Development Plan (2018YFC0116904), National Nature Science Foundation of China under Grant (61971413, 61801476), Jiangsu Key Technology Research Development Program (BE2018610), Jiangsu Natural Science Foundation (BK20192004, BK20180221), Shandong Natural Science Foundation (ZR2020QF018, ZR2020QF019, and ZR2020QF022), Suzhou Municipal Health and Family Planning Commission's Key Diseases Diagnosis and Treatment Program (LCZX201930), Suzhou Science \& Technology Projects for People's Livelihood 
(SS202054, SS202065, SS201866), Jinan Innovation Team, Quancheng 5150 Project, Lishui Medical Key Discipline Construction Project (2020ZDYF09, 2017ZDXK09), Suzhou Health Science \& Technology Project (2020Q003),and Suzhou Health Personnel Training Program.

Availability of data and materials

The datasets used and/or analyzed during the current study are available from the corresponding author on reasonable request.

\section{Ethics approval and consent to participate}

The study is approved by the Ethics Committee of the Third Affiliated Hospital of Soochow University.

\section{Consent for publication}

All subjects gave written informed consent in accordance with the Declaration of Helsinki.

\section{Competing interests}

The authors declare that they have no competing interests.

\section{Author details}

${ }^{1}$ Suzhou Institute of Biomedical Engineering and Technology, Chinese Academy of Sciences, Suzhou, China. ${ }^{2}$ Suzhou Key Laboratory of Medical and Health Information Technology, Suzhou, China. ${ }^{3}$ Jinan Guoke Medical Engineering Technology Development Co., LTD, Jinan, China. ${ }^{4}$ Department of Pediatrics, The Third Affiliated Hospital of Soochow University, Changzhou, China. ${ }^{5}$ Trauma Center, Khandwa District Hospital, Khandwa, India. ${ }^{6}$ Department of Clinical Psychology, The Third Affiliated Hospital of Soochow University, Changzhou, China.

Received: 11 August 2020 Accepted: 23 January 2021

Published online: 12 February 2021

\section{References}

1. Saiphoo AN, Halevi LD, Vahedi Z. Social networking site use and self-esteem: a meta-analytic review. Personal Individ Differ. 2020;153:109639.

2. Rieger S, Göllner R, Trautwein U, et al. Low self-esteem prospectively predicts depression in the transition to young adulthood: a replication of Orth, Robins, and Roberts. J Pers Soc Psychol. 2016;110(1):e16.

3. Chavez RS, Heatherton TF. Structural integrity of frontostriatal connections predicts longitudinal changes in selfesteem. Soc Neurosci. 2017;12(3):280-6.

4. Zilverstand A, Huang AS, Alia-Klein N, et al. Neuroimaging impaired response inhibition and salience attribution in human drug addiction: a systematic review. Neuron. 2018;98(5):886-903.

5. Wang $Y$, Zhang $L$, Kong $X$, et al. Pathway to neural resilience: Self-esteem buffers against deleterious effects of poverty on the hippocampus. Hum Brain Mapp. 2016;37(11):3757-66.

6. Lieberman MD, Straccia MA, Meyer ML, et al. Social, self,(situational), and affective processes in medial prefrontal cortex (MPFC): Causal, multivariate, and reverse inference evidence. Neurosci Biobehav Rev. 2019;99:311-28.

7. Bede P, Hardiman O. Longitudinal structural changes in ALS: a three time-point imaging study of white and gray matter degeneration. Amyotroph Later Scler Frontotemporal Degener. 2018;19(3-4):232-41.

8. Solé-Casals J, Serra-Grabulosa JM, Romero-Garcia R, et al. Structural brain network of gifted children has a more integrated and versatile topology. Brain Struct Funct. 2019;224(7):2373-83.

9. Kelley WM, Macrae CN, Wyland CL, et al. Finding the self? An event-related fMRI study. J Cogn Neurosci. 2002;14(5):785-94.

10. Goldin P, Ziv M, Jazaieri H, et al. Randomized controlled trial of mindfulness-based stress reduction versus aerobic exercise: effects on the self-referential brain network in social anxiety disorder. Front Hum Neurosci. 2012;6:295.

11. Cuingnet R, Gerardin E, Tessieras J, et al. Automatic classification of patients with Alzheimer's disease from structural MRI: a comparison of ten methods using the ADNI database. Neuroimage. 2011;56(2):766-81.

12. Schmitgen MM, Niedtfeld I, Schmitt R, et al. Individualized treatment response prediction of dialectical behavior therapy for borderline personality disorder using multimodal magnetic resonance imaging. Brain Behav. 2019;9(9):e01384.

13. Erol RY, Orth U. Self-esteem development from age 14 to 30 years: a longitudinal study. J Pers Soc Psychol. 2011;101(3):607.

14. Peng B, Saxena A, Wang S, et al. Enhancing the representation of multiple anatomical network for young adults with self-esteem difference. In: 2019 12th international congress on image and signal processing, BioMedical Engineering and Informatics (CISP-BMEI). IEEE. 2019;1-5.

15. Cheng W, Rolls ET, Qiu J, et al. Functional connectivity of the precuneus in unmedicated patients with depression. Biol Psychiatry Cogn Neurosci Neuroimaging. 2018;3(12):1040-9.

16. Yang J, Xu X, Chen $Y$, et al. Trait self-esteem and neural activities related to self-evaluation and social feedback. Sci Rep. 2016;6(1):1-10.

17. Van Schie CC, Chiu CD, Rombouts SARB, et al. When compliments do not hit but critiques do: an fMRI study into self-esteem and self-knowledge in processing social feedback. Soc Cogn Affect Neurosci. 2018;13(4):404-17

18. García JA, y Olmos FC, Matheu ML, et al. Self esteem levels vs global scores on the Rosenberg self-esteem scale. Heliyon. 2019;5(3):e01378.

19. Thirion JP. Image matching as a diffusion process: an analogy with Maxwell's demons. Med Image Anal. 1998;2:243-60.

20. Smith SM. Fast robust automated brain extraction. Hum Brain Mapp. 2002;17(3):143-55. 
21. Wang L, Shi F, Li G, et al. 4D segmentation of brain MR images with constrained cortical thickness variation. PLoS ONE. 2013;8(7):e64207.

22. Tzourio-Mazoyer N, Landeau B, Papathanassiou D, et al. Automated anatomical labeling of activations in SPM using a macroscopic anatomical parcellation of the MNI MRI single-subject brain. Neuroimage. 2002;15(1):273-89.

23. Li G, Wang L, Yap PT, et al. Computational neuroanatomy of baby brains: a review. Neurolmage. 2019:185:906-25.

24. Peng $H$, Xie P, Liu L, et al. Brain-wide single neuron reconstruction reveals morphological diversity in molecularly defined striatal, thalamic, cortical and claustral neuron types. bioRxiv. 2020;675280.

25. Guyon I, Weston J, Barnhill S, et al. Gene selection for cancer classification using support vector machines. Mach Learn. 2002;46(1-3):389-422.

26. Peng B, Lu J, Saxena A, et al. Examining brain morphometry associated with self-esteem in young adults using multilevel-ROI-features-based classification method. Front Comput Neurosci. 2017;11:37.

\section{Publisher's Note}

Springer Nature remains neutral with regard to jurisdictional claims in published maps and institutional affiliations.

- fast, convenient online submission

- thorough peer review by experienced researchers in your field

- rapid publication on acceptance

- support for research data, including large and complex data types

- gold Open Access which fosters wider collaboration and increased citations

- maximum visibility for your research: over 100M website views per year

At BMC, research is always in progress.

Learn more biomedcentral.com/submissions 